
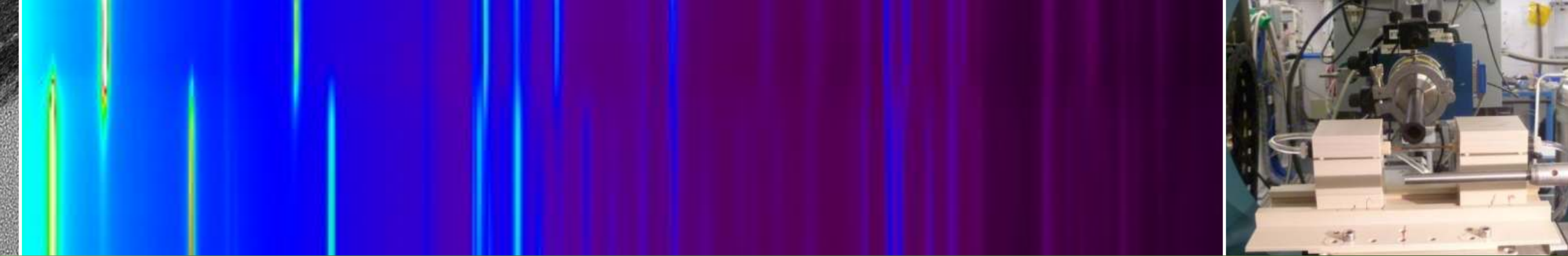

\title{
Ion exchange in Fougerite: influence of crystal size and structural defects
}

Myriam l. Agnel, Sylvain Grangeon, François Fauth, Erik Elkaïm, Francis Claret, Marjorie Roulet, Fabienne Warmont, Christophe Tournassat
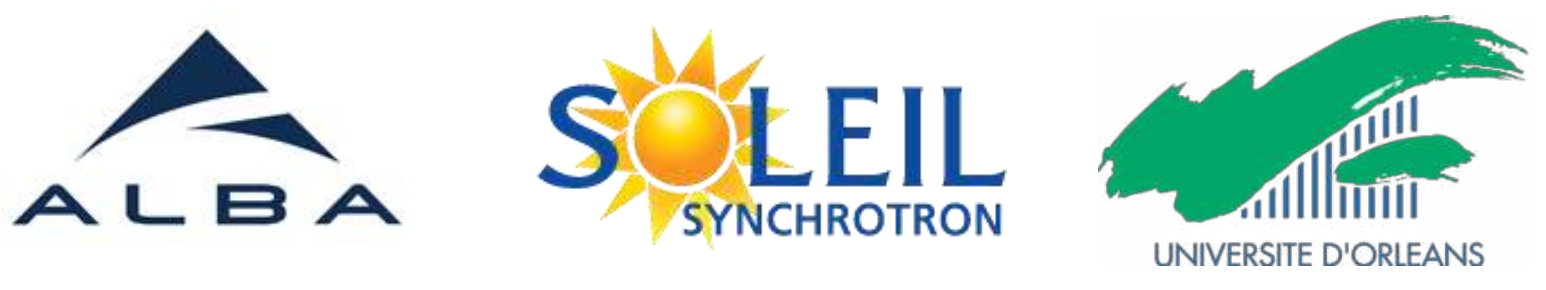


\section{Introduction}

WORLD SOIL RESOURCES
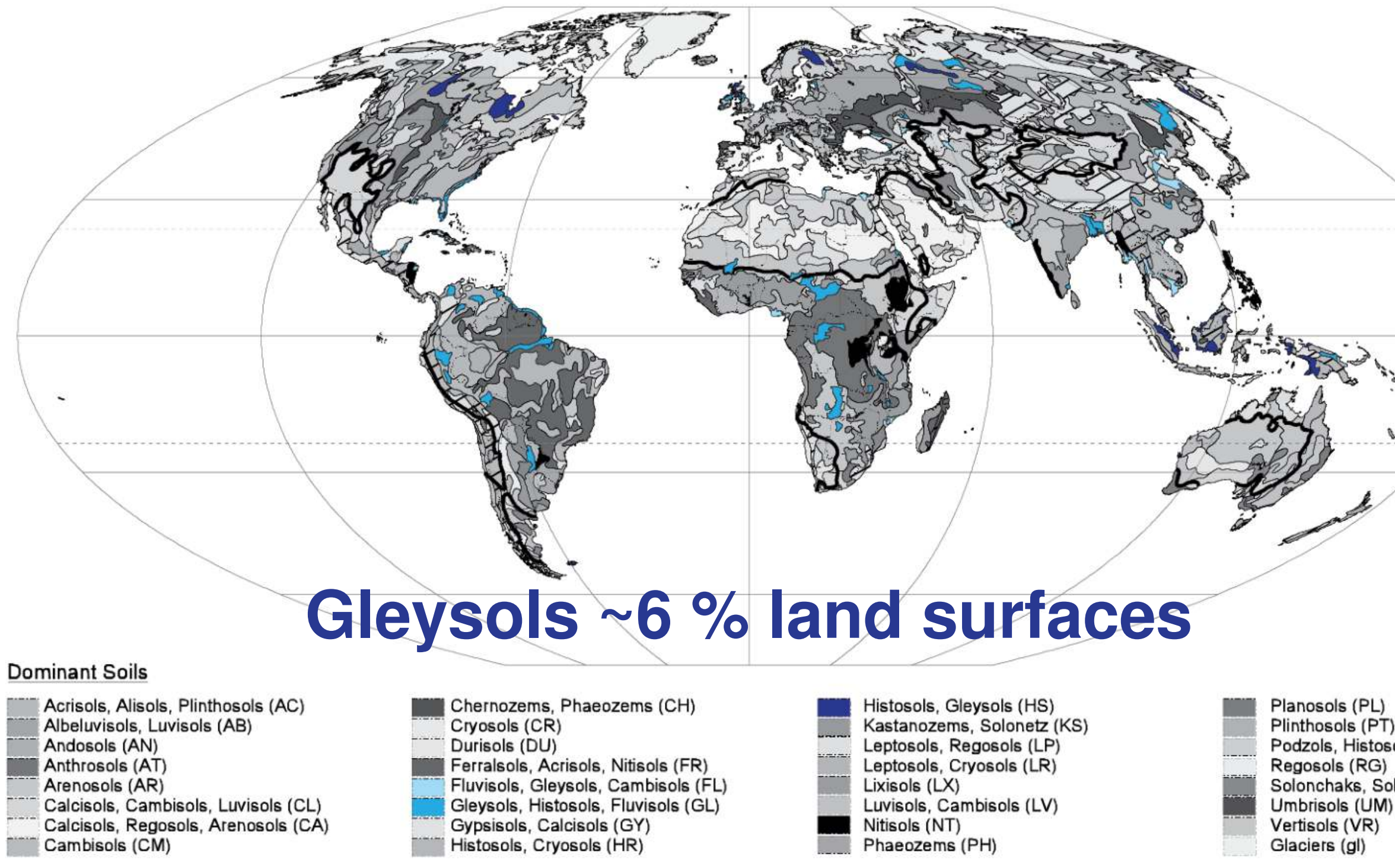


\section{Introduction}

Gleysols $\rightarrow$ Minerals capable of sorbing anions $\rightarrow$ for ex. Fougerite

$\rightarrow$ Phenomena of sorption/desorption

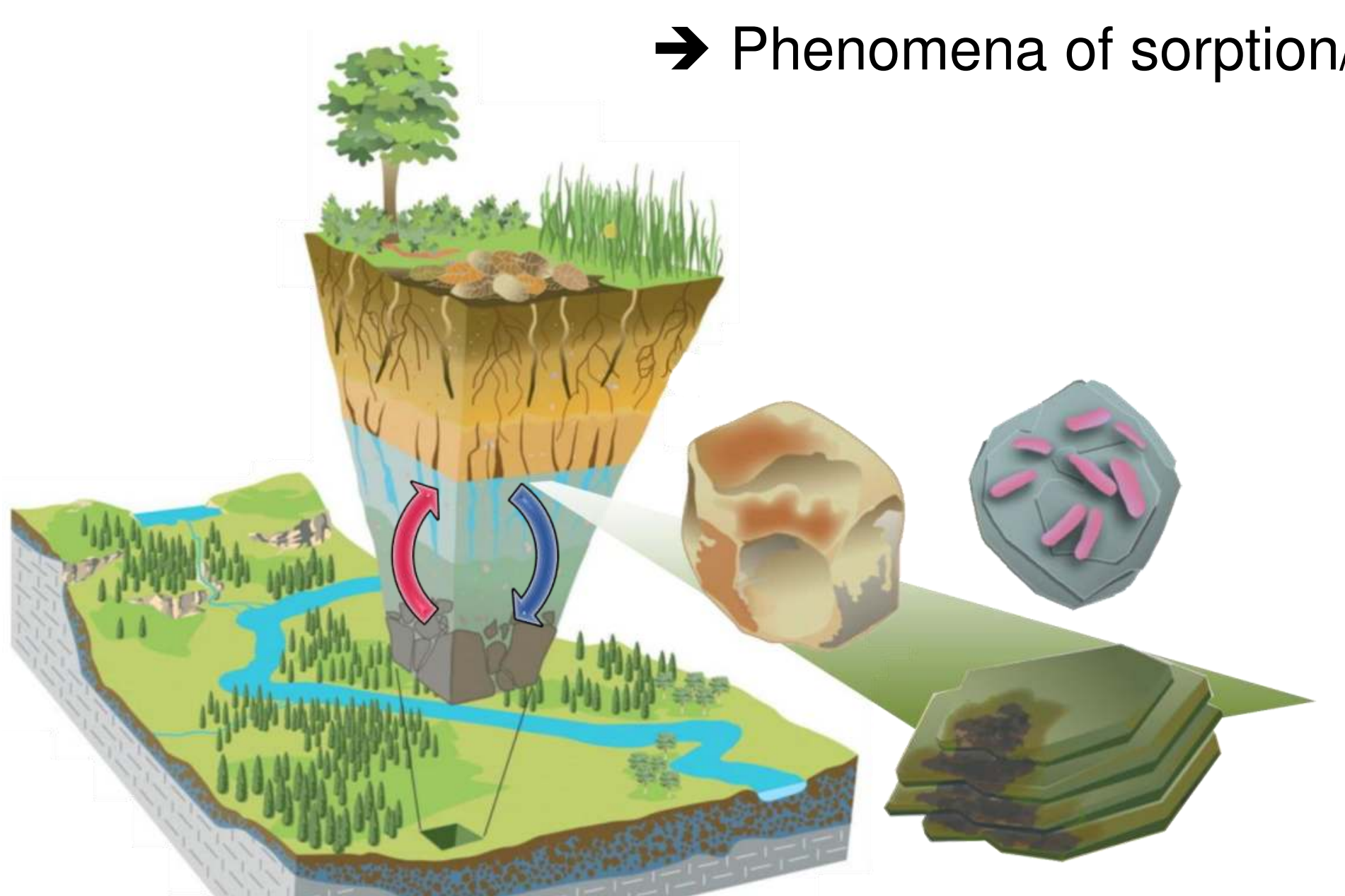

Cycle of elements 


\section{Introduction}

\section{Fougerite:}

- 1996: Discovered in gleysols

- Already known as an artificial phase found in iron pipes: Green rust

- Layered double hydroxide

- Exchangeable anion in the interlayer

$\left[\mathrm{Fe}^{2+}{ }_{1-x} \mathrm{Fe}^{3+}{ }_{x}(\mathrm{OH})_{2}\right]^{+x}\left[\mathrm{x} / \mathrm{n} \mathrm{A}^{-\mathrm{n}} \cdot \mathrm{mH}_{2} \mathrm{O}\right]^{-x}$

$$
\left(\mathrm{A}=\mathrm{OH}^{-}, \mathrm{Cl}^{-}, \mathrm{CO}_{3}^{2-}, \mathrm{SO}_{4}{ }^{2-} \ldots\right)
$$

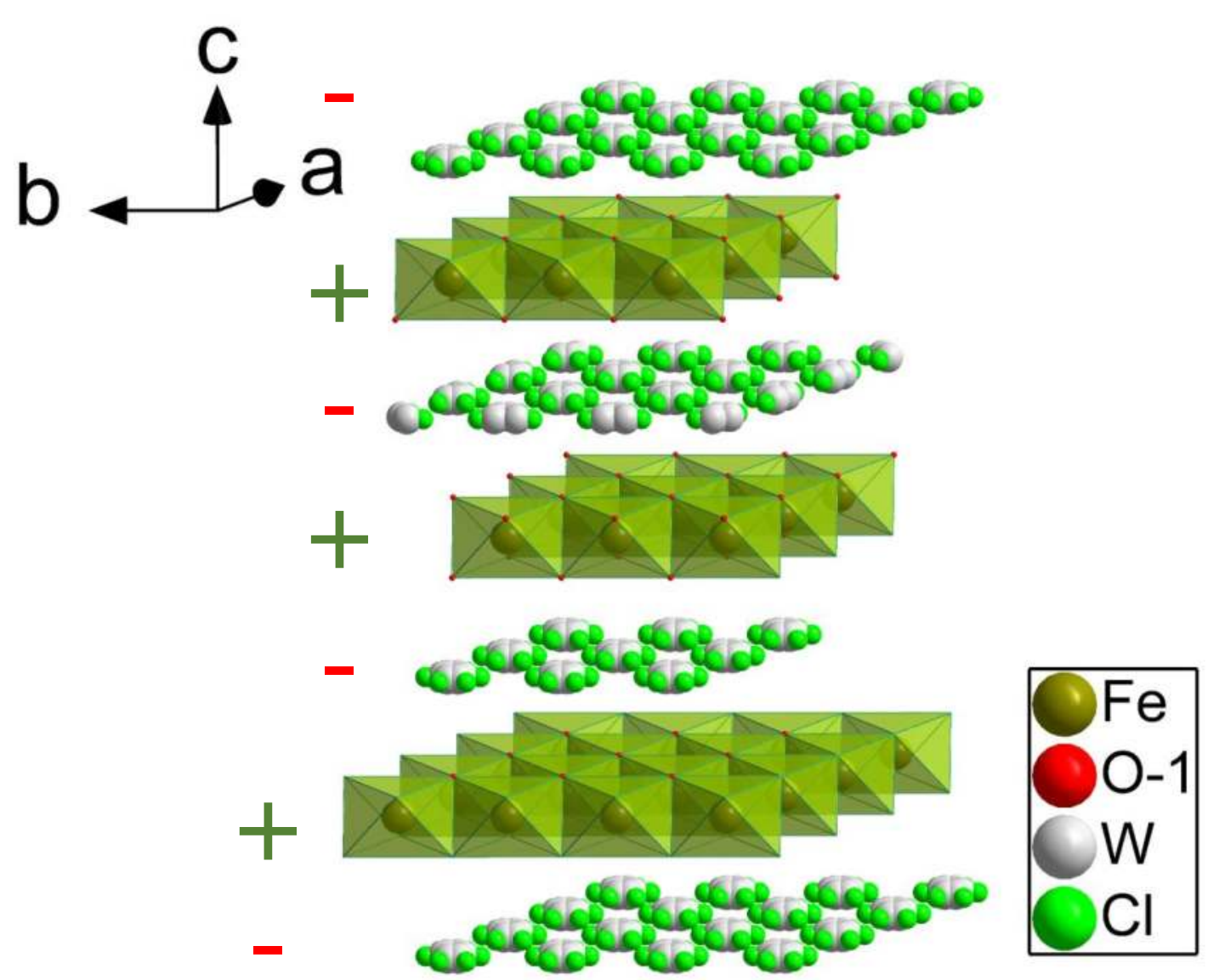




\section{Objectives}

\section{Previously ...}

Input

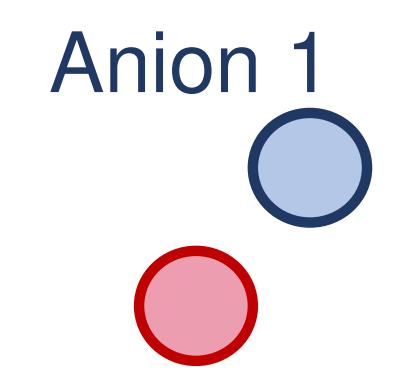

Anion 2

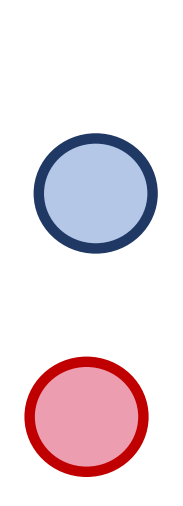

0
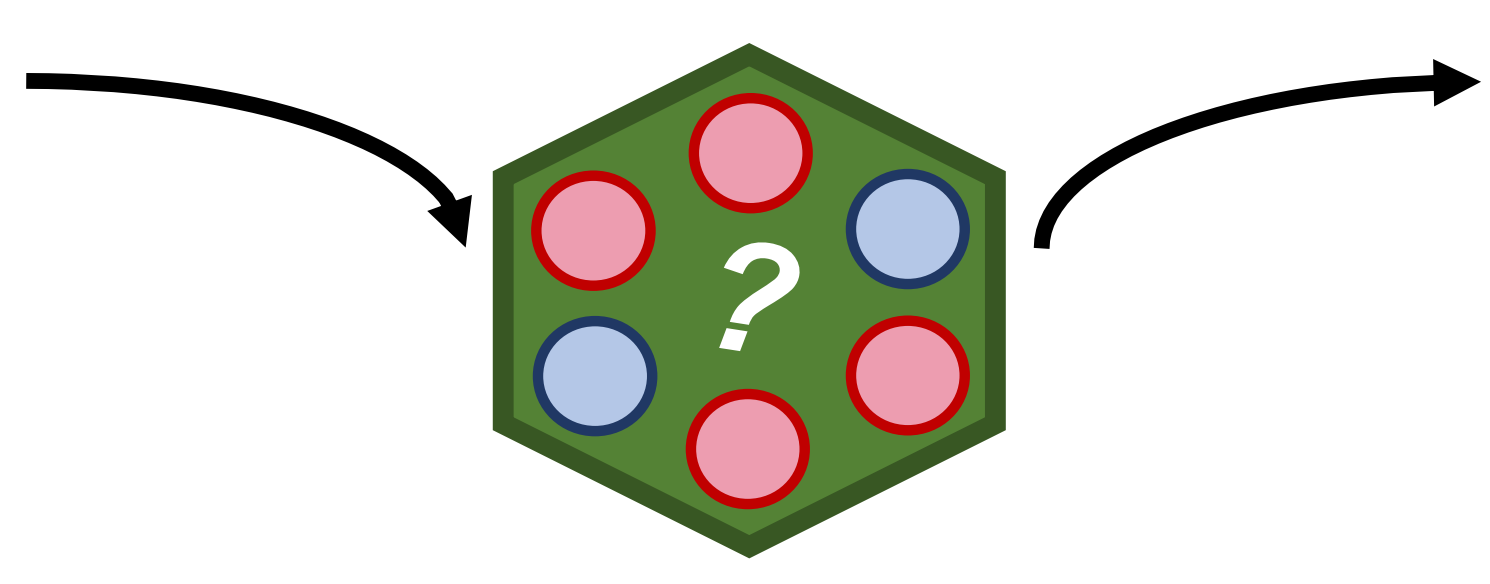

$\because$

Output

GR

Affinity : $\mathrm{CO}_{3}{ }^{2-}>\mathrm{SO}_{4}{ }^{2-}$ and $\mathrm{OH}^{-}>\mathrm{Cl}^{-}>\mathrm{NO}_{3}{ }^{-}$

Question:

What is the mechanism of ion exchange in green rusts \& how can we quantify it?

- Discrete phase dissolution-precipitation

- Solid solution or lon exchange 


\section{Studied samples - Structure}

\section{Micro-GR}

$\rightarrow$ Fougerite

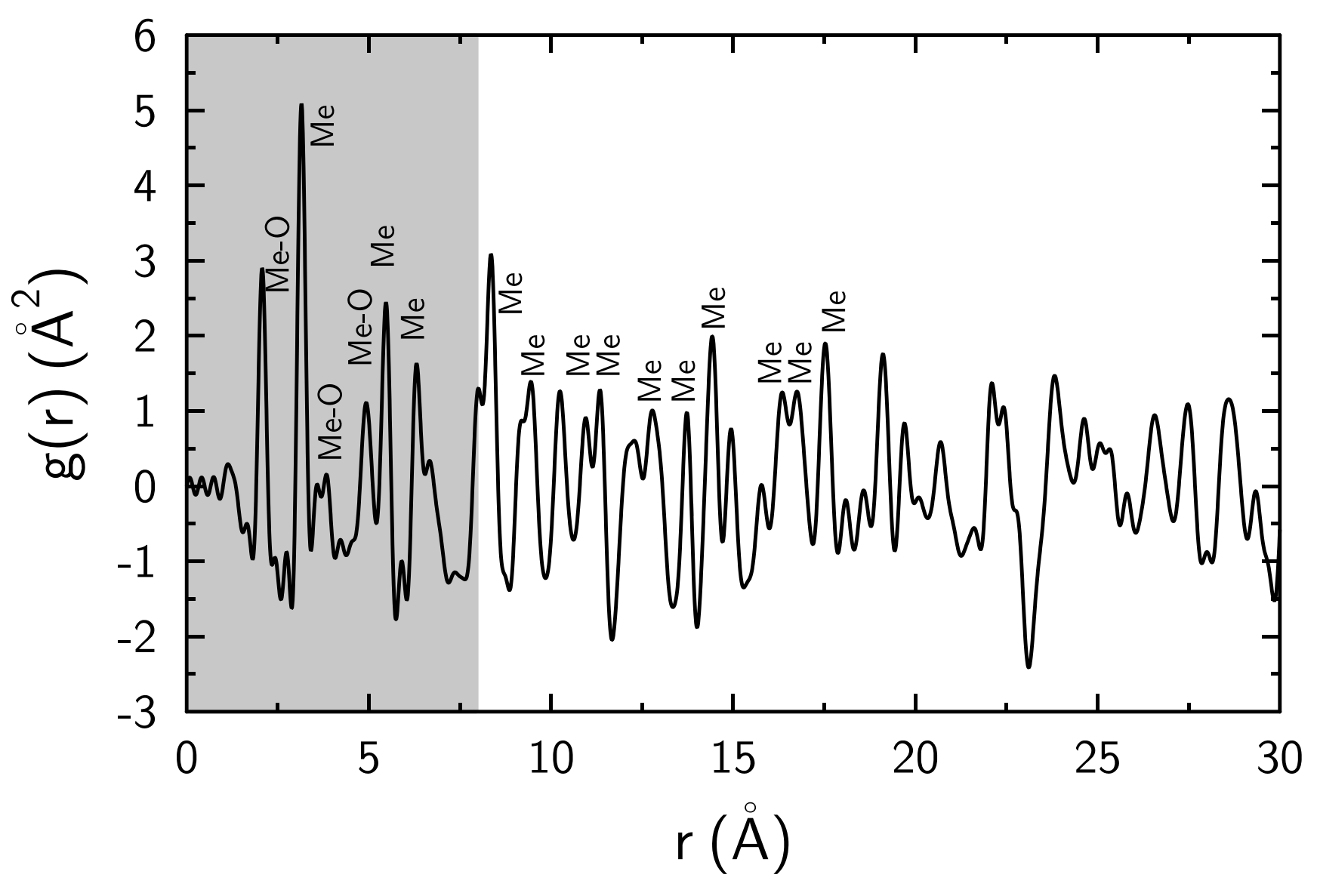

\section{S, LEIL}

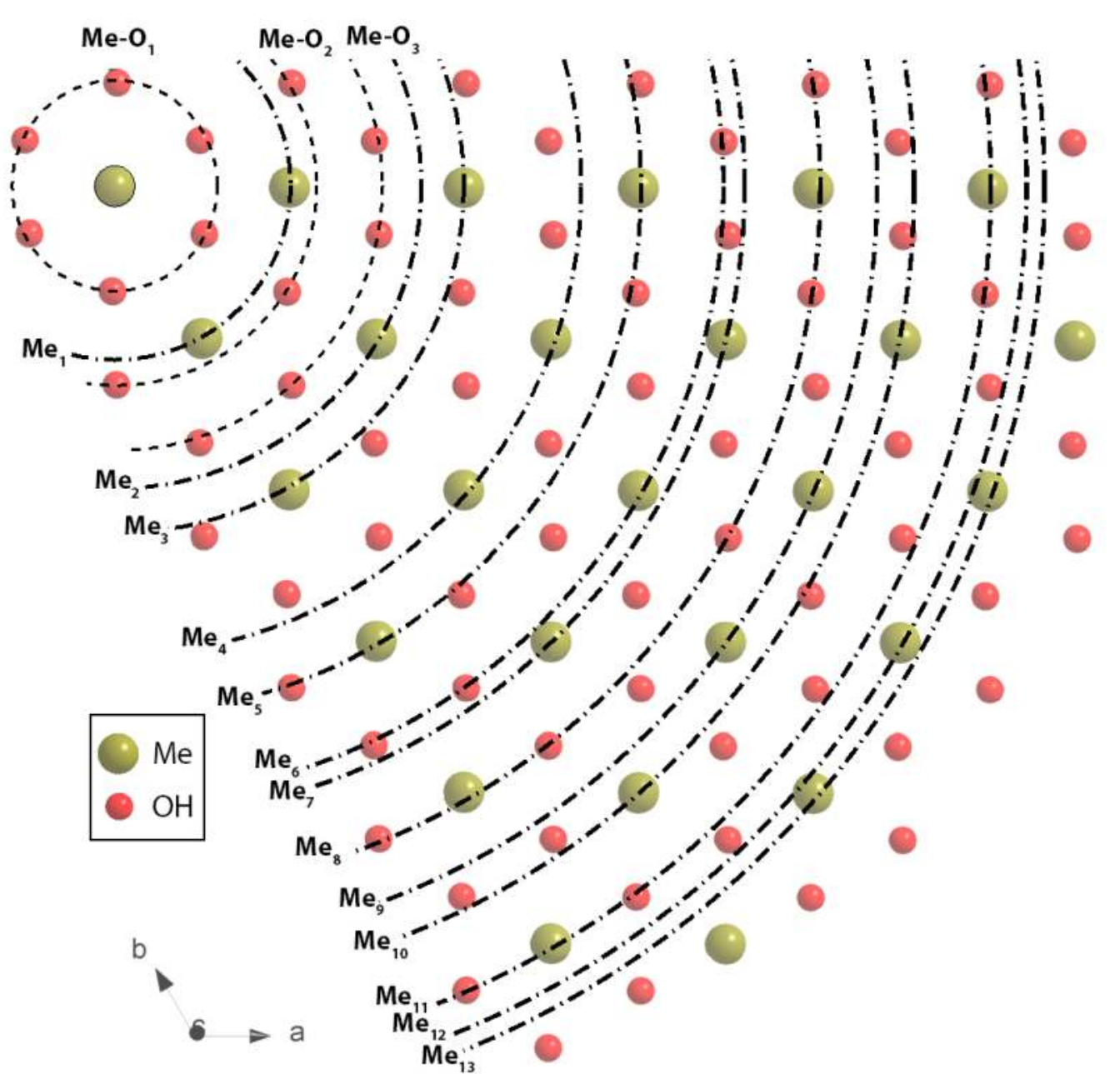




\section{Studied samples - Structure}

\section{Micro-GR}

Fougerite

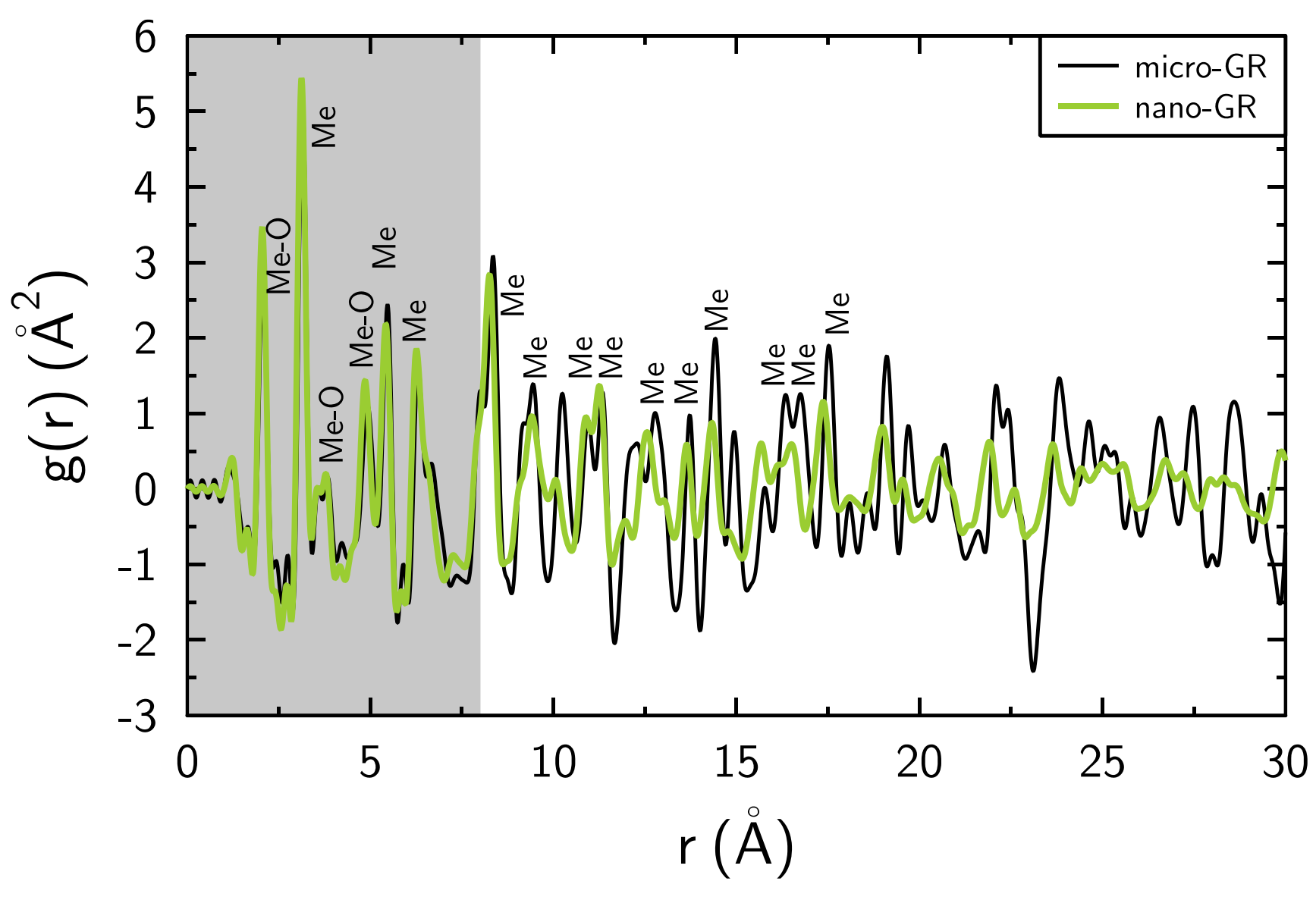

\section{Nano-GR}

$\rightarrow$ Layer similar to Fougerite $\rightarrow$ Stacking faults?

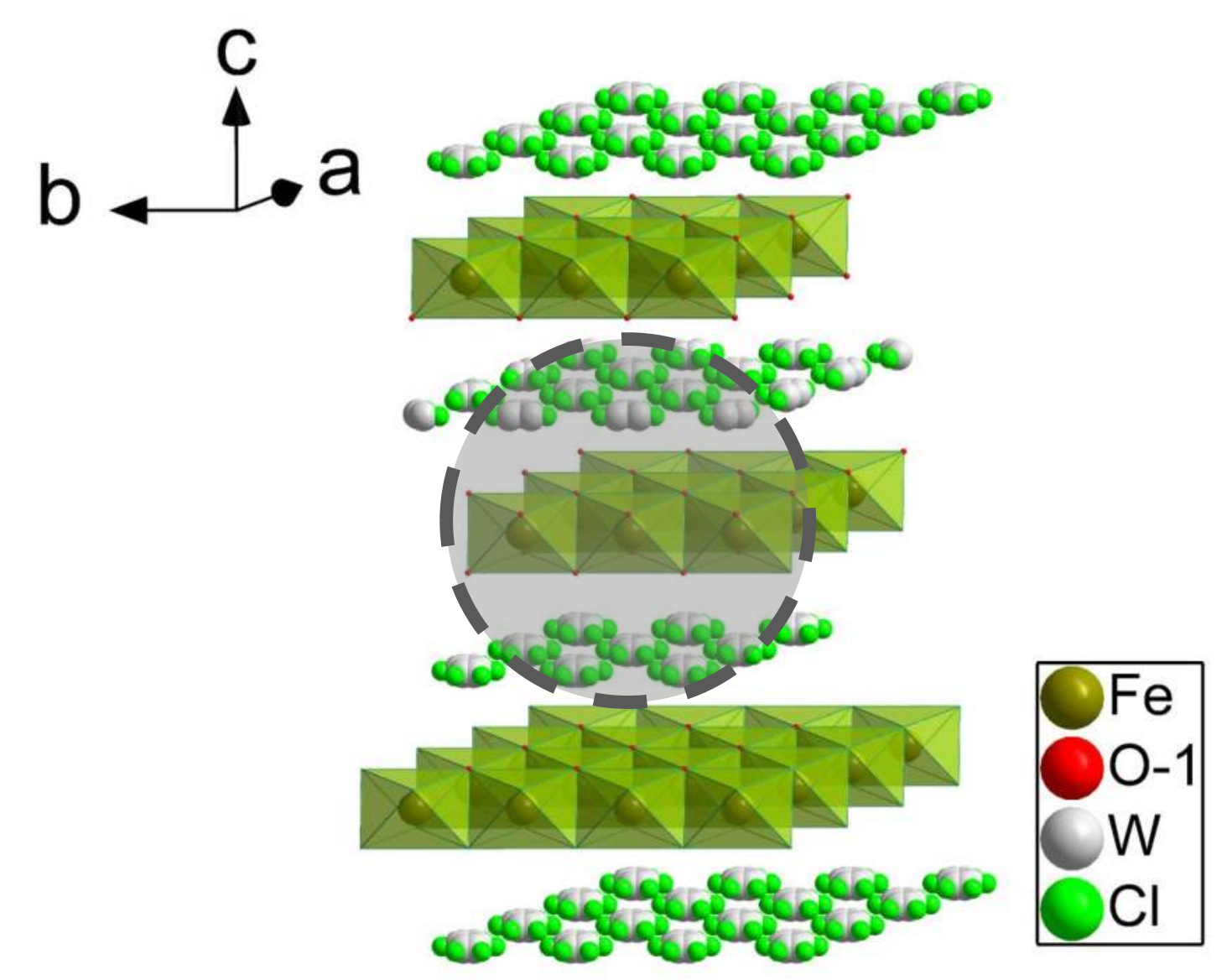




\section{Micro-GR}

Fougerite $\rightarrow 0.5-1 \mu \mathrm{m}$

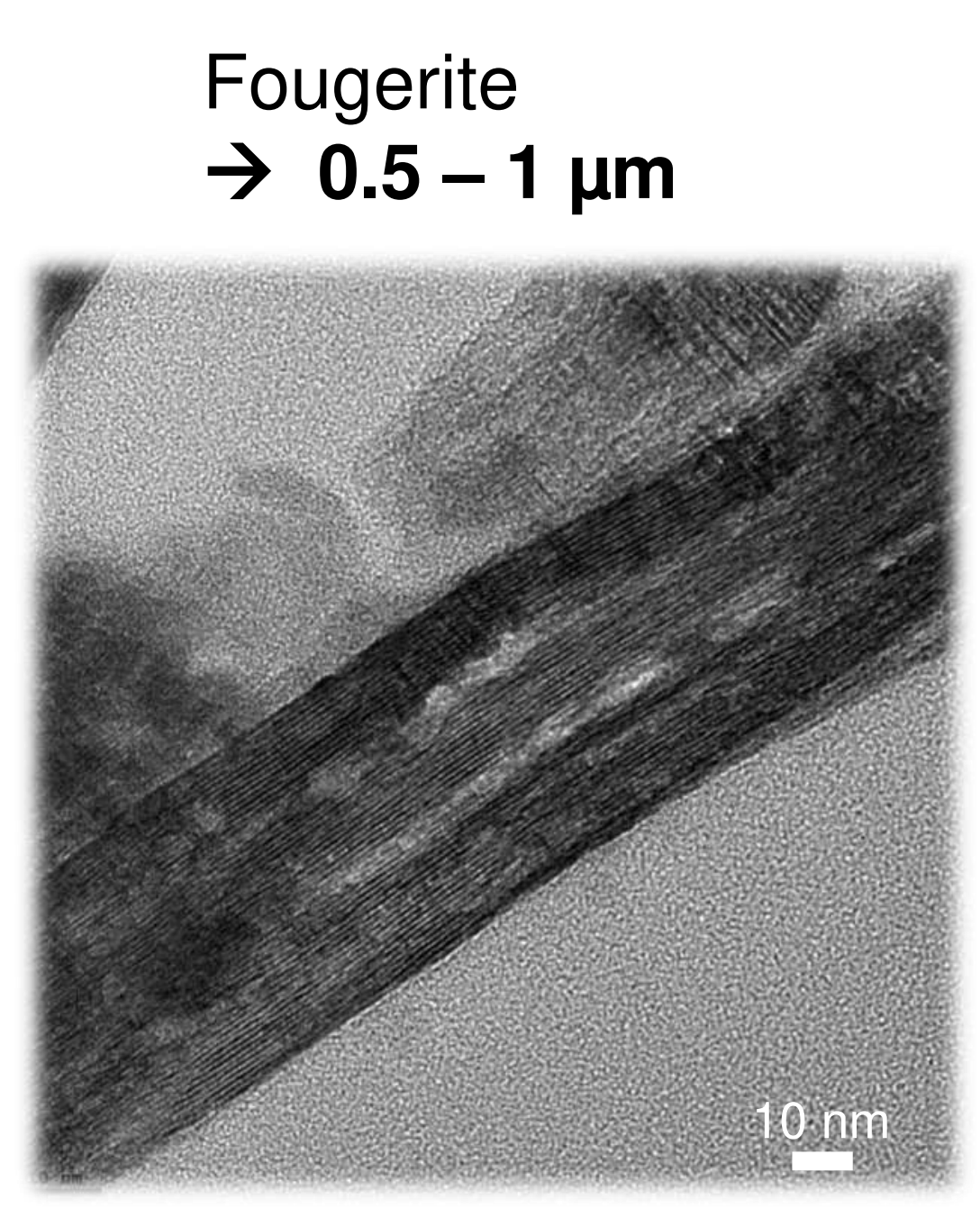

\section{Studied samples - Structure}

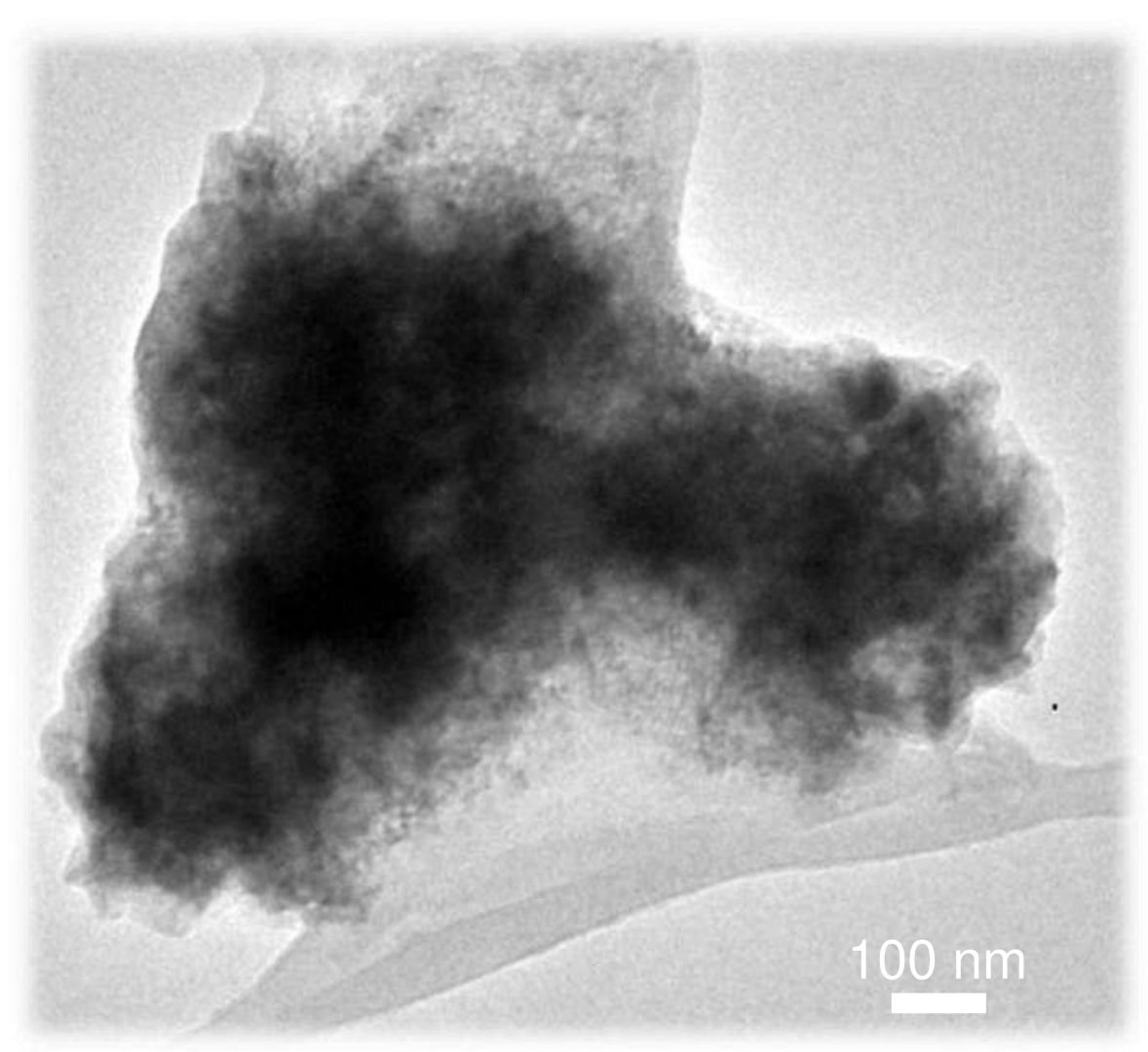

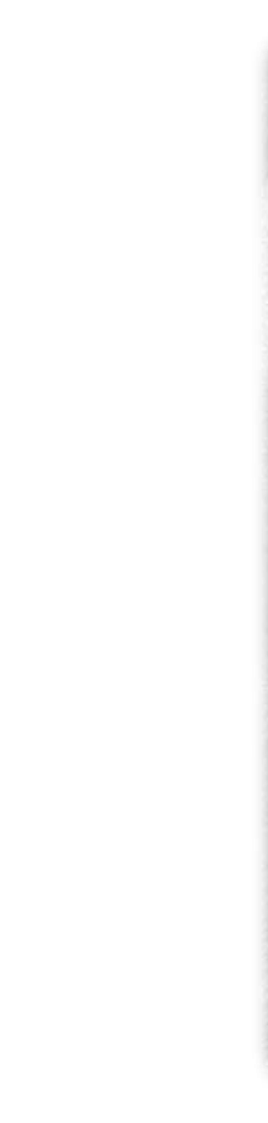
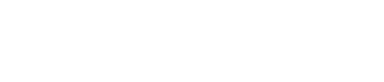

\section{Nano-GR \\ Layer similar to Fougerite $\rightarrow \mathbf{1 0}-\mathbf{3 0} \mathrm{nm}$} $100 \mathrm{nn}$

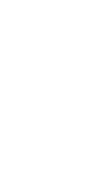

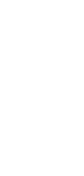

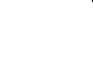

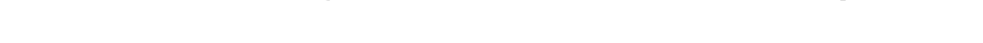

烈




\section{Studied samples - Structure}

\section{Micro-GR}

$\rightarrow$ 3D-ordered Fougerite

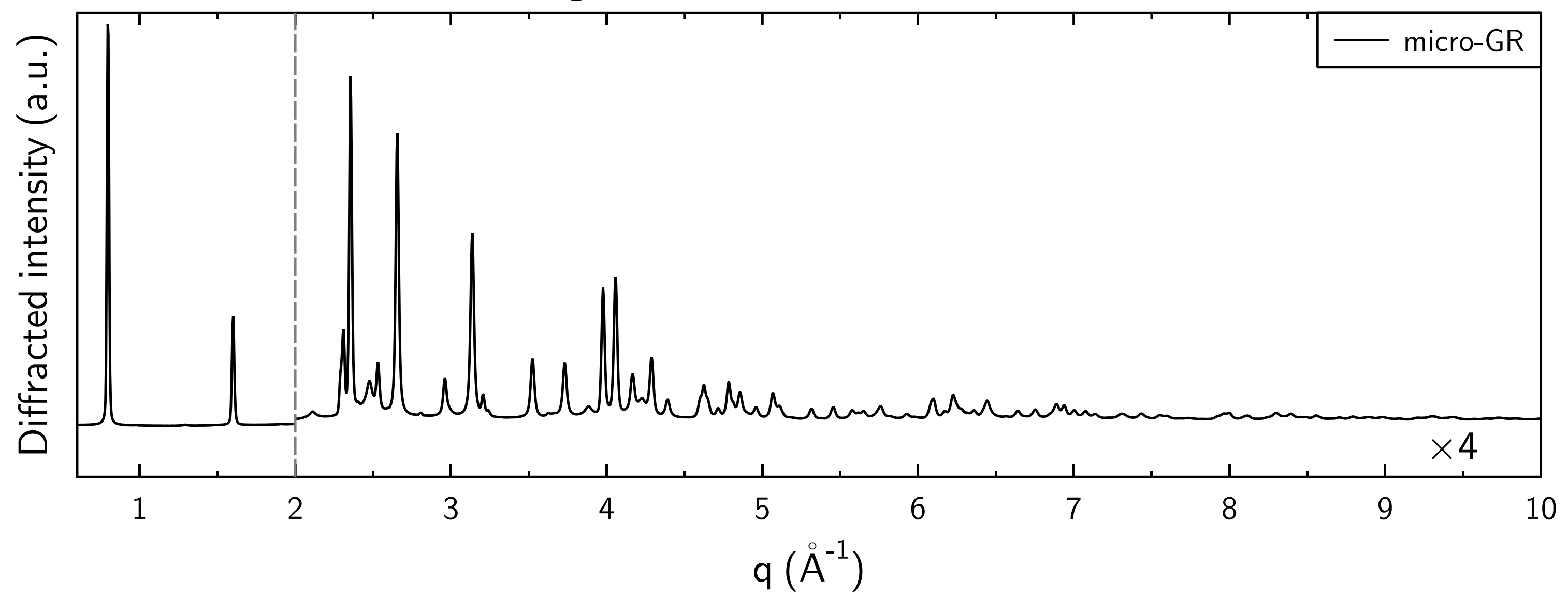




\section{Studied samples - Structure}

\section{Micro-GR}

\section{Nano-GR}

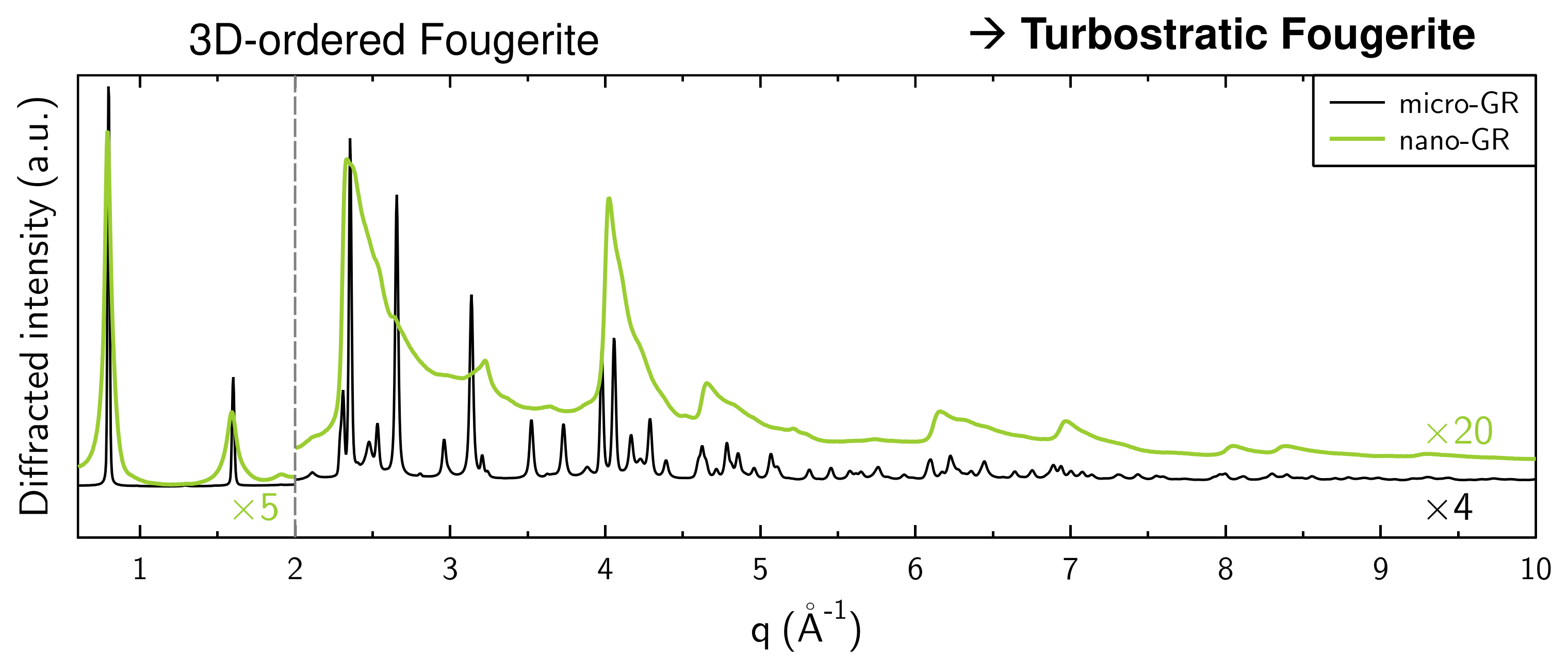




\section{Transformation from $\mathrm{GR}(\mathrm{Cl})$ to $\mathrm{GR}\left(\mathrm{SO}_{4}\right)$}

\section{- Exchange mechanism}

Synchrotron in situ and time resolved X-ray diffraction

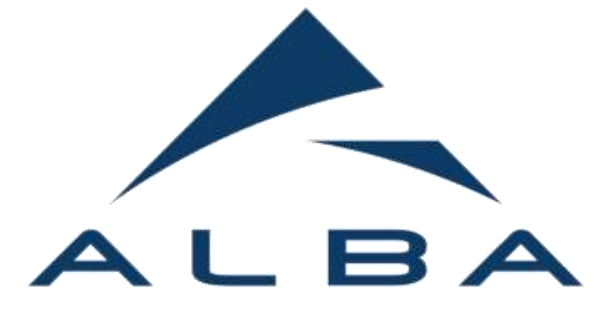

GR powder

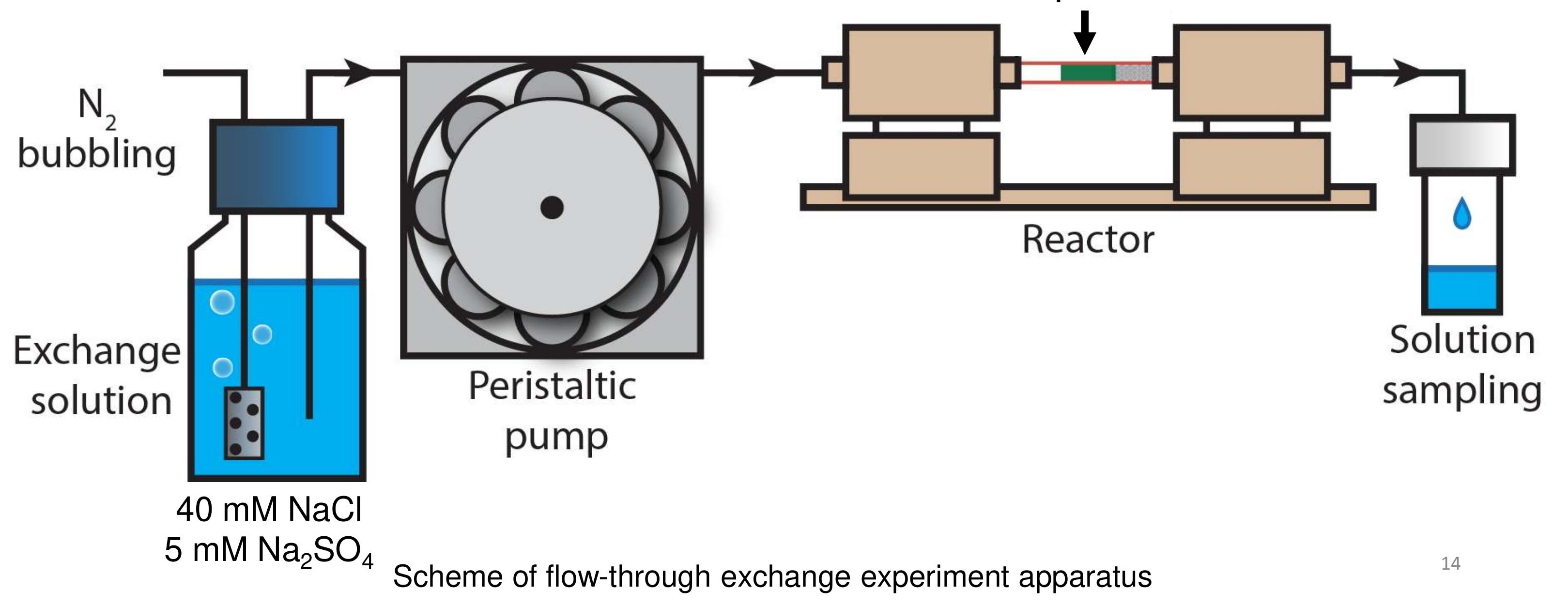




\section{Transformation from $\mathrm{GR}(\mathrm{Cl})$ to $\mathrm{GR}\left(\mathrm{SO}_{4}\right)$}

\section{- Exchange mechanism}

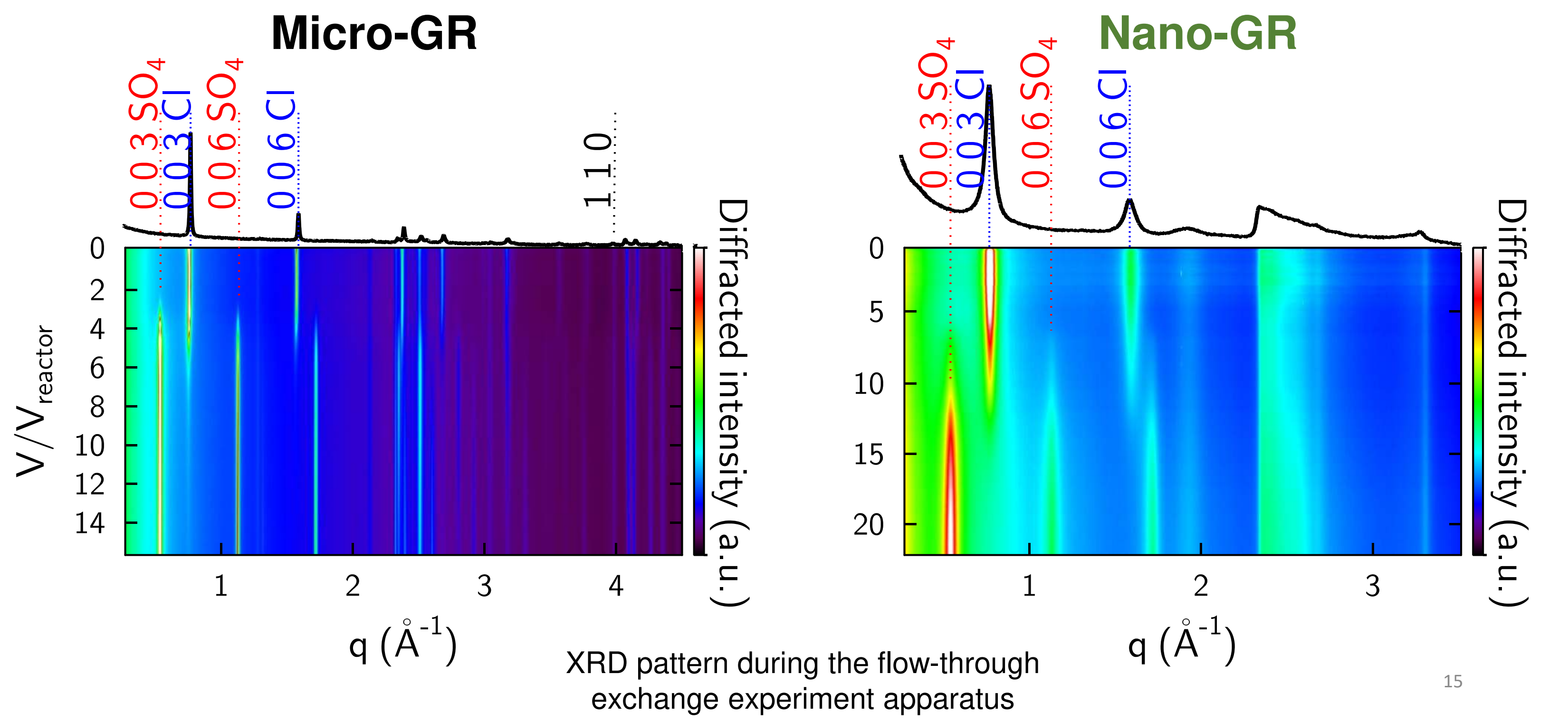




\section{Transformation from $\mathrm{GR}(\mathrm{Cl})$ to $\mathrm{GR}\left(\mathrm{SO}_{4}\right)$}

\section{- Exchange mechanism}

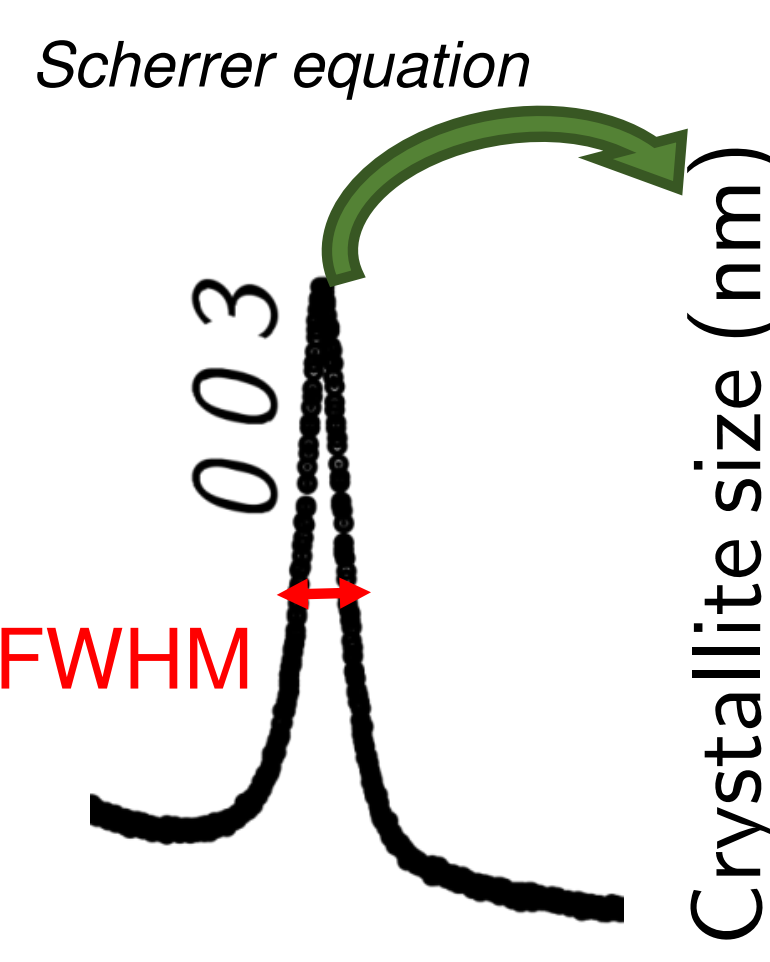

Diffraction peak
Micro-GR

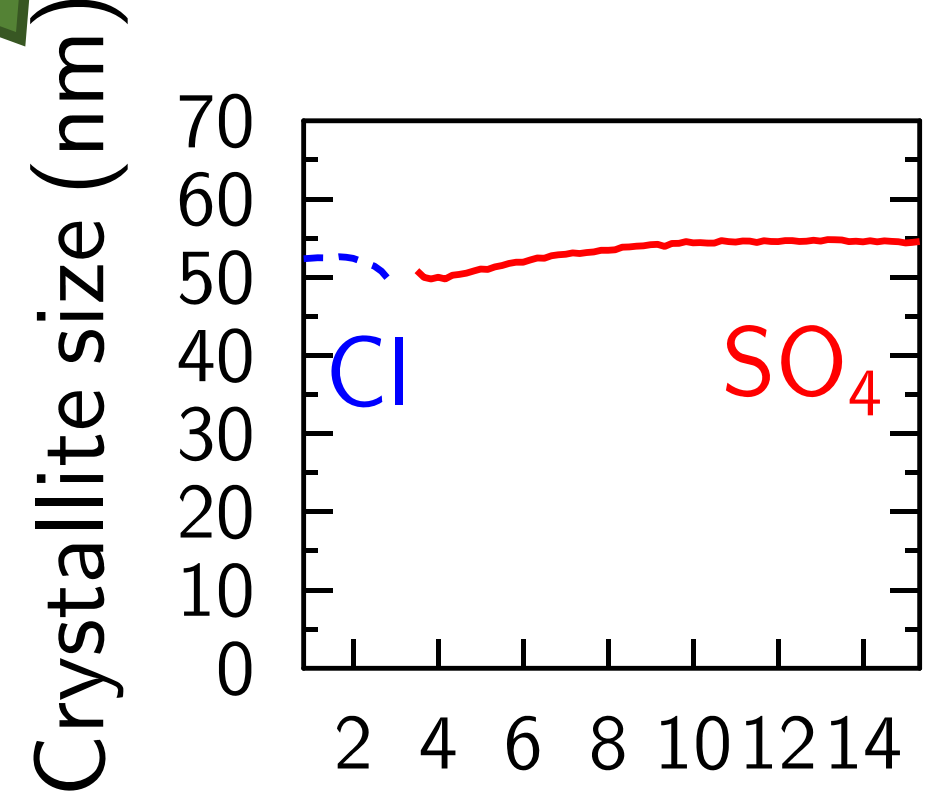

$\mathrm{V} / \mathrm{V}_{\text {reactor }}$
Nano-GR

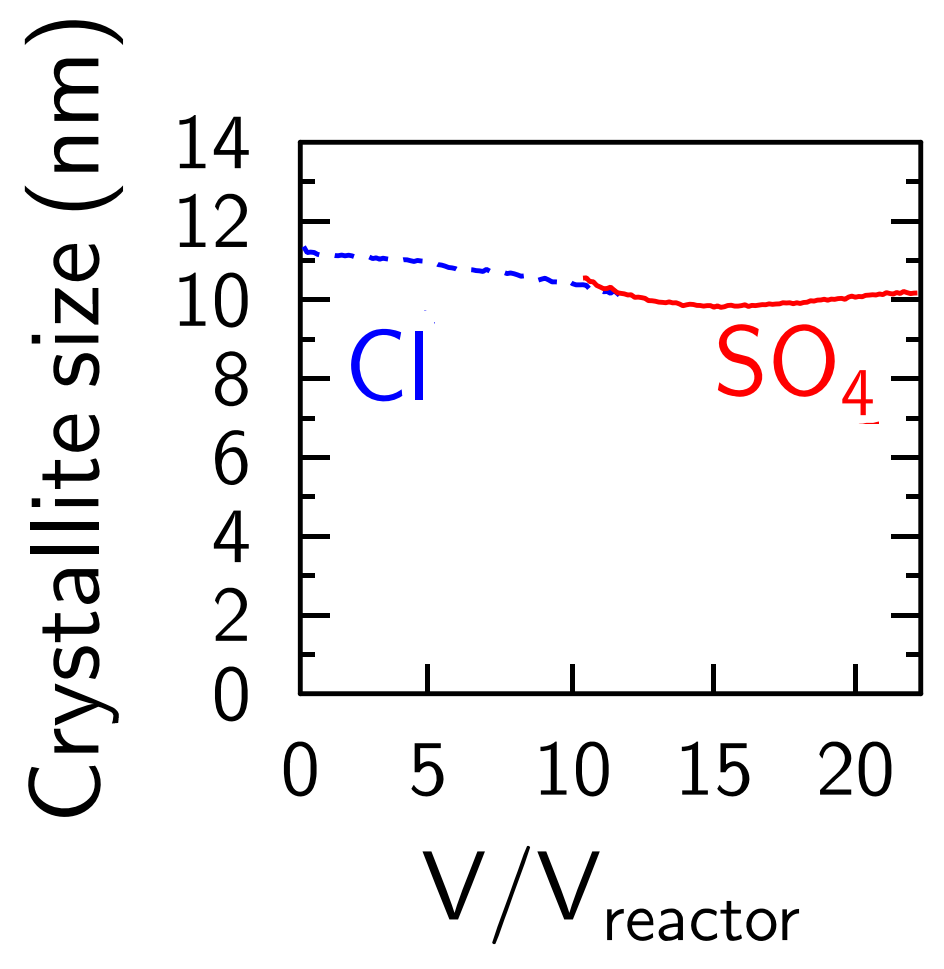

Crystallite size inferred from $\mathrm{FWHM}$ of $003 \mathrm{Cl}$ 


\section{Transformation from $\mathrm{GR}(\mathrm{Cl})$ to $\mathrm{GR}\left(\mathrm{SO}_{4}\right)$}

\section{- Exchange mechanism}

Micro-GR

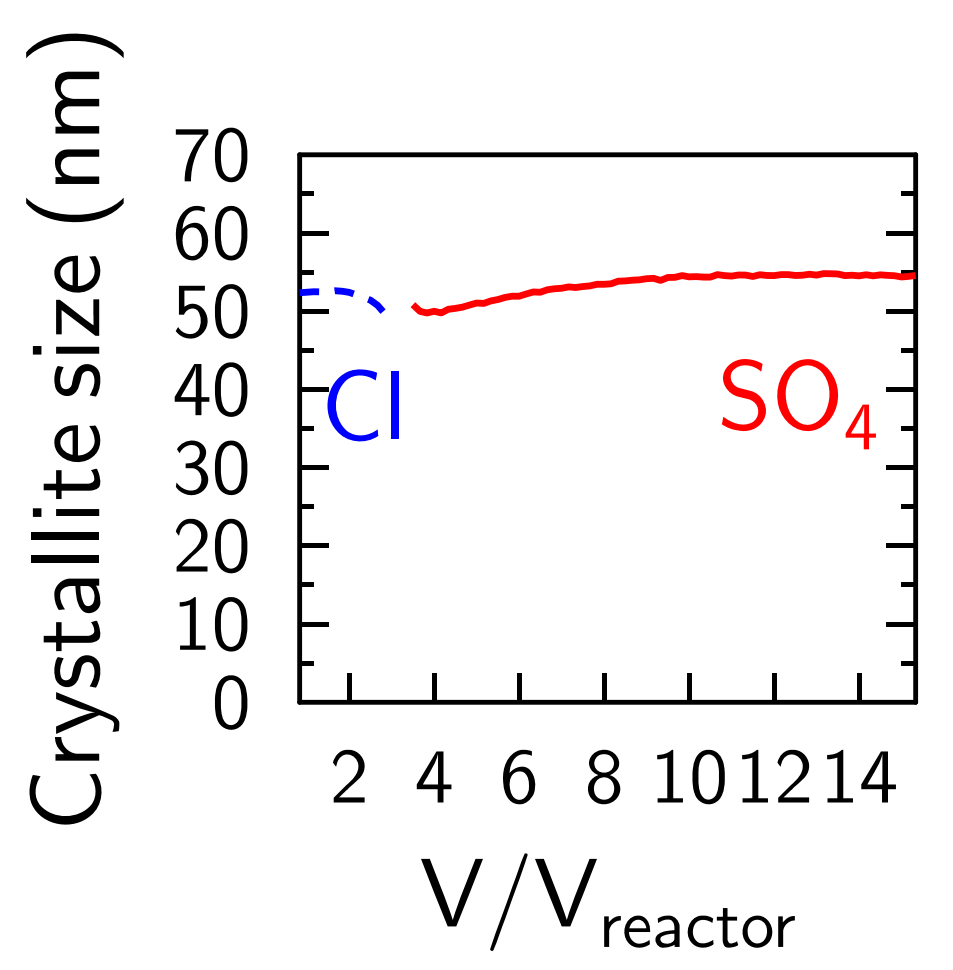

Nano-GR

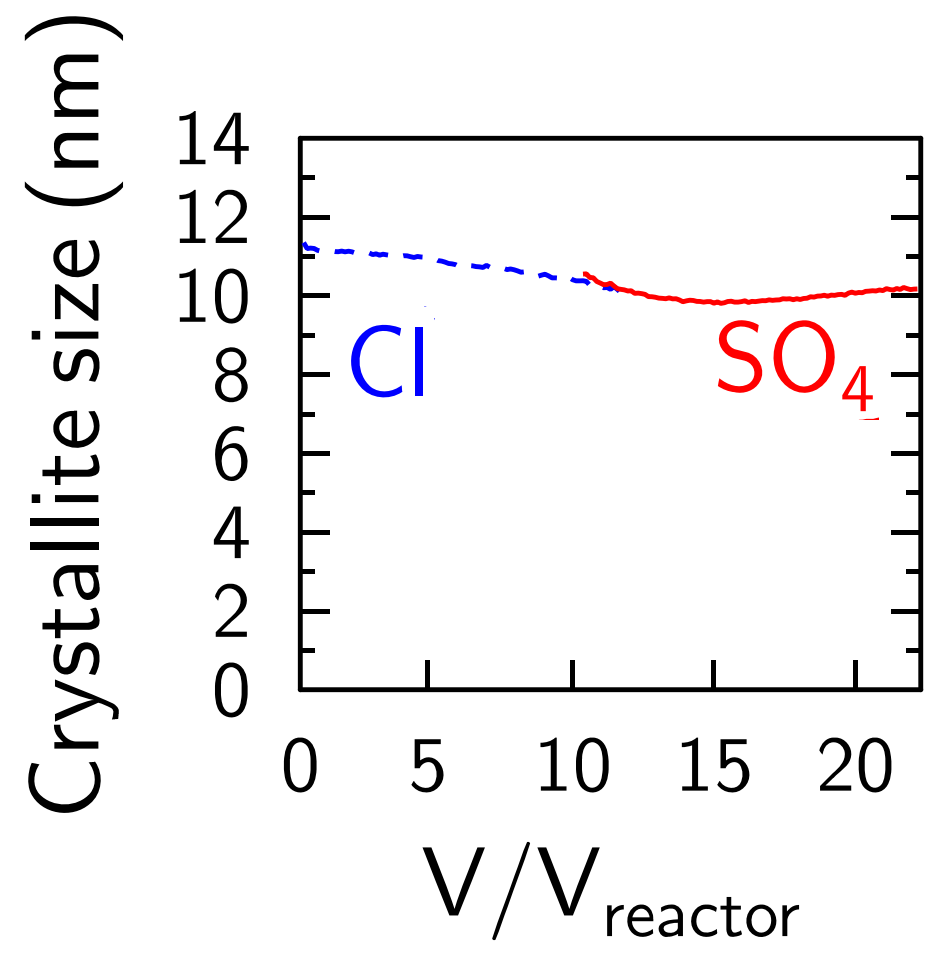

$\rightarrow$ Constant crystallite size

Exchange mechanism (no precipitation/dissolution, no phase transformation) 


\section{Transformation from $\mathrm{GR}(\mathrm{Cl})$ to $\mathrm{GR}\left(\mathrm{SO}_{4}\right)$}

\section{- Exchange model}

Batch experiment with various concentrations:

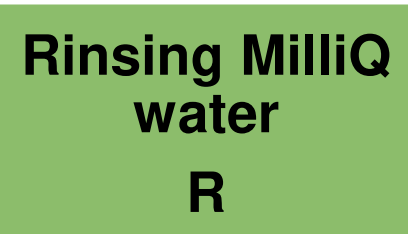

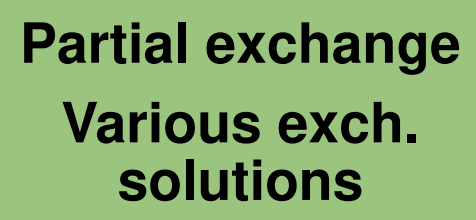
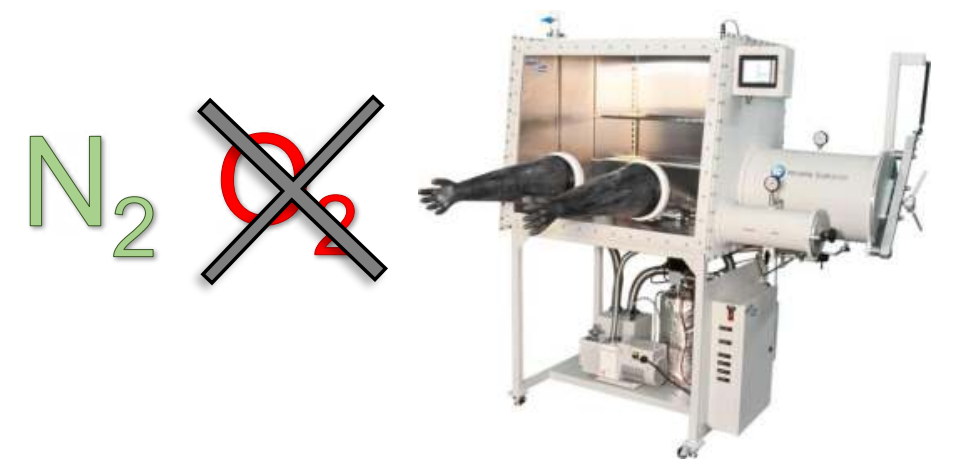

Rinsing MilliQ

water

R
Total exchange $25 \mathrm{mM} \mathrm{Na}_{2} \mathrm{SO}_{4}$

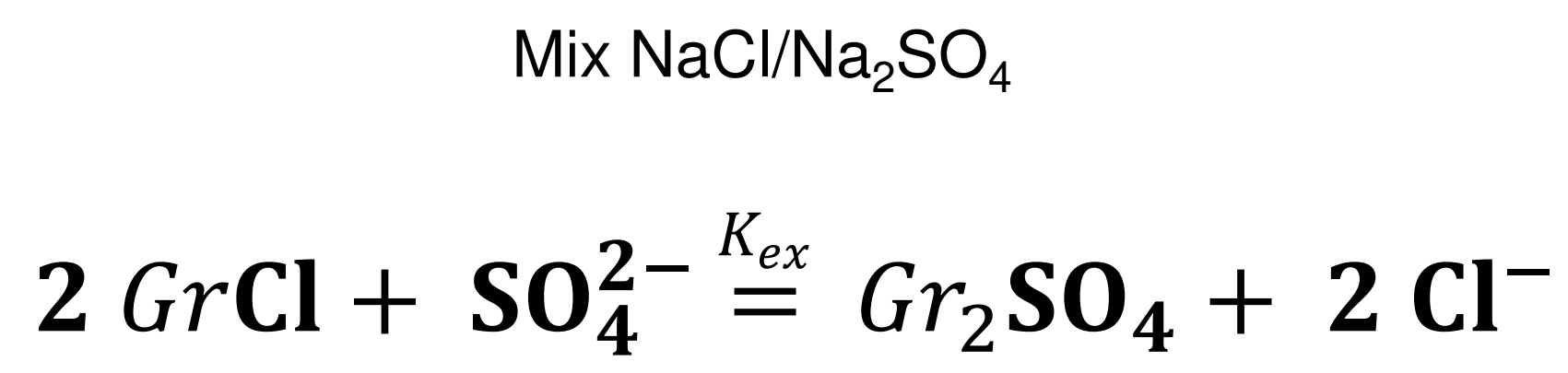

Anion Exchange Capacity:

$\rightarrow$ Micro-GR : 2 mol $/ \mathrm{kg}_{\text {anhydrous GR }}$

$\rightarrow$ Nano-GR : $1.6 \mathrm{~mol}_{\mathrm{c}} / \mathrm{kg}_{\text {anhydrous GR }}$

Smectite CEC: $0.8-1.5 \mathrm{~mol} / \mathrm{kg}$ Kaolinite CEC: $0.03-0.15 \mathrm{~mol} / \mathrm{kg}$ 


\section{Transformation from $\mathrm{GR}(\mathrm{Cl})$ to $\mathrm{GR}\left(\mathrm{SO}_{4}\right)$}

\section{- Exchange model}

Micro-GR
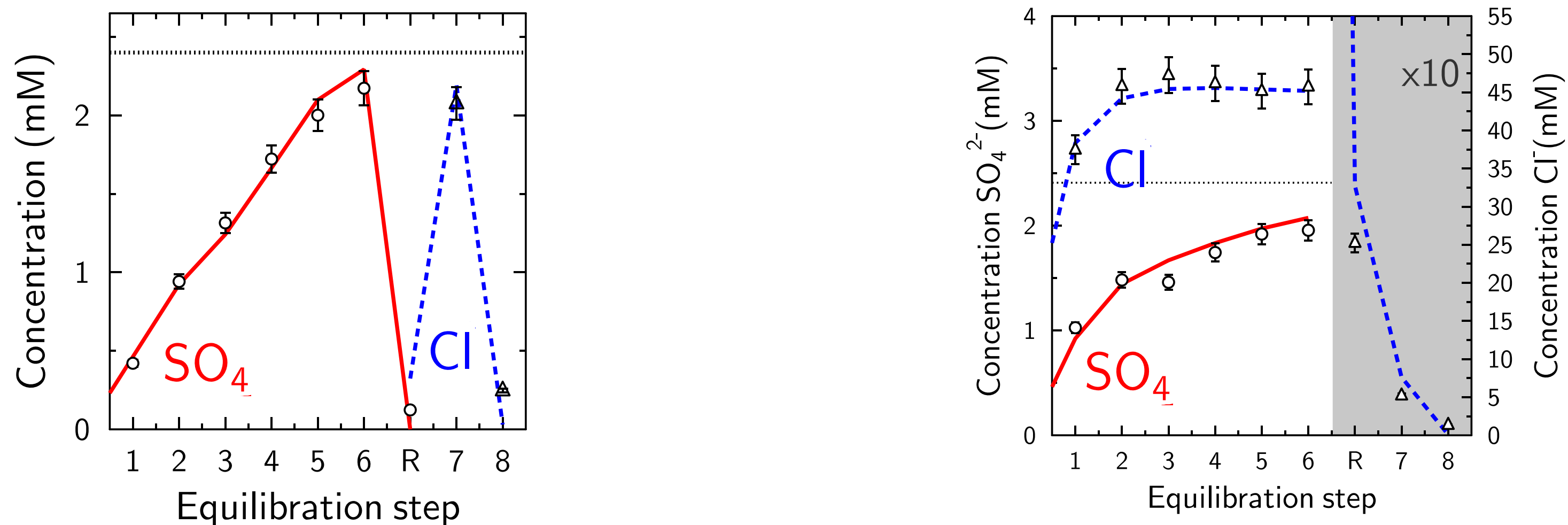

Batch experiment results with $2.5 \mathrm{mM} \mathrm{Na}_{2} \mathrm{SO}_{4} / 45 \mathrm{mM} \mathrm{NaCl}$

Exchange convention of Rothmund-Kornfeld

$$
\beta=2.4 \log K_{\text {ex }}^{R K}=0.3 \quad \beta=5 \log K_{\text {ex }}^{R K}=0.2
$$


What is the mechanism of ion exchange in green rusts \& how can we quantify it?

\section{Structure:}

$\checkmark$ Simple exchange mechanism (no dissolution/recrystallization)

$\checkmark$ Independent of crystal size or stacking defects

$\checkmark$ Swelling from 8 to $11 \AA$ after exchange with $\mathrm{SO}_{4}{ }^{2-}$

\section{Thermodynamic:}

$\checkmark \quad \checkmark 2 \mathrm{Cl}^{-}: 1 \mathrm{SO}_{4}{ }^{2-}$ exchange $\rightarrow$ higher affinity for $\mathrm{SO}_{4}{ }^{2-}$

$\checkmark$ Fast exchange

$\checkmark$ Model with exchange convention of Rothmund \& Kornfeld

$\rightarrow$ Parameters dependent on stacking or size 


\section{Thank you for your attention!}

Acknowledgments:
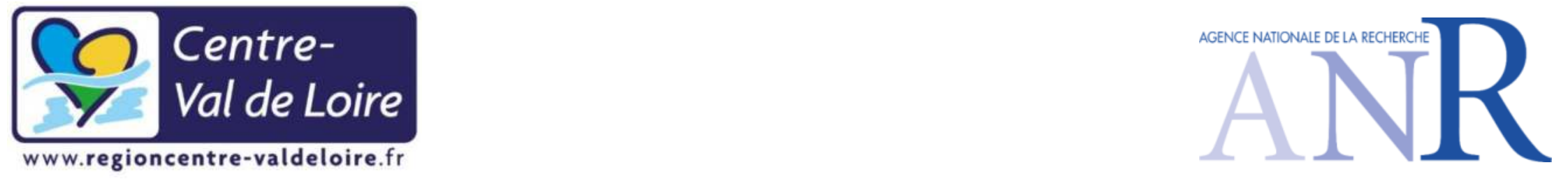

For more information, article:

Agnel, M.I.; Grangeon, S.; Fauth, F.; Elkaïm, E.; Claret, F.; Roulet, M.; Warmont, F.; Tournassat, C. Mechanistic and thermodynamic insights into anion exchange by green rust. 2020, 54, 851-861.

\section{For any questions, please contact me at myriam.agnel@cnrs-orleans.fr}

\section{References:}

Chorover, J.; Kretzschmar, R.; Garcia-Pichel, F.; Sparks, D. L. Soil Biogeochemical Processes within the Critical Zone. Elements 2007, 3 (5), $321-326$.

Hadi, J.; Grangeon, S.; Warmont, F.; Seron, A.; Greneche, J. M. A Novel and Easy Chemical-Clock Synthesis of Nanocrystalline Iron-Cobalt Bearing Layered Double Hydroxides. J. Colloid Interface Sci. 2014, 434, 130-140.

Trolard, F.; Abdelmoula, M.; Bourrié, G.; Humbert, B.; Génin, J.-M. R. Mise En Évidence d'un Constituant de Type «rouilles Vertes» Dans Les Sols Hydromorphes. Proposition de l'existence d'un Nouveau Minéral : La «Fougerite». Comptes rendus l'Académie des Sci. Série 2. Sci. la terre des planètes 1996, 323 (12), $1015-1022$.

Trolard, F.; Bourrié, G.; Abdelmoula, M.; Refait, P.; Feder, F. Fougerite, a New Mineral of the Pyroaurite-lowaite Group: Description and Crystal Structure. Clays Clay Miner, 2007, 55 (3), 323-334. 


\section{SI - lon exchange}

\section{$2 \mathrm{ACl}+\mathrm{SO}_{4}^{2-}=\mathrm{A}_{2} \mathrm{SO}_{4}+2 \mathrm{Cl}^{-}$}

Equilibrium constant:

$$
K_{e x}=\frac{\left(A_{2} S_{4}\right)\left(C l^{-}\right)^{2}}{(A C l)^{2}\left(S_{4}^{2-}\right)}
$$

Equivalent fraction:

$$
E_{C l}=\frac{[\mathrm{ACl}]}{[\mathrm{ACl}]+2\left[\mathrm{~A}_{2} \mathrm{SO}_{4}\right]} \quad E_{\mathrm{SO}_{4}}=\frac{2\left[\mathrm{~A}_{2} \mathrm{SO}_{4}\right]}{[\mathrm{ACl}]+2\left[\mathrm{~A}_{2} \mathrm{SO}_{4}\right]}
$$

Selectivity coefficient:

Gaines \& Thomas:

$$
K_{e x, G T}=\frac{\left(C l^{-}\right)^{2}}{\left(S_{4}^{2-}\right)} \times \frac{E_{S O_{4}}}{E_{C l}^{2}}
$$

Rothmund-Kornfeld:

$$
K_{e x, R K}=\frac{\left(\mathrm{Cl}^{-}\right)^{2}}{\left(\mathrm{SO}_{4}^{2-}\right)}\left(\frac{E_{\mathrm{SO} 4}}{E_{\mathrm{Cl}}{ }^{2}}\right)^{1 / \beta}
$$

